\title{
Un mini pressiomètre pour essais sur modèles réduits centrifugés
}

\author{
O. Beckerich, J. Garnier, G. Rault \\ Laboratoire Central des Ponts et Chaussées \\ D. Levacher \\ Université du Havre
}

\begin{abstract}
Geotechnical studies on centrifuged small scale models require more and more soil characterizations during centrifugation. Currently used equipments alow to reproduce cone and vane tests at model scale. The miniaturization of the pressuremeter, which is the most complete in-situ test, is in progress at the Nantes LCPC center. The prototype probe is at a $1 / 20^{\text {th }}$ scale from the Menard probe and its expansion is similar. The first results are promising: the curves and pressuremeter profiles are well represented, and correlations between in-situ parameters are satisfied.
\end{abstract}

\section{Introduction}

\subsection{La reconnaissance géotechnique}

Les essais in situ de reconnaissance géotechnique les plus menés en Europe sont l'essai au pénétromètre statique ou dynamique (en première approche) et l'essai pressiométrique. Ce dernier présente deux avantages principaux :

- il peut être réalisé dans tous les types de sol.

- il est le seul à fournir une relation contrainte-déformation du sol, caractérisée par le module pressiométrique Em et la pression limite pl.

La fiabilité des résultats, à la condition du strict respect du mode opératoire, en a fait l'un des essais les plus répandus pour le gimensionnement des ouvrages les plus importants. Il est également à la base des règles françaises de dimensionnement de fondations superficielles et profondes.

\subsection{La modélisation physique en centrifugeuse}

Pour les grands ouvrages aux caractéristiques peu communes, une voie privilégiée de l'étude est la modélisation physique.

Le respect des conditions de similitude, nécessaire à une reproduction correcte du mécanisme, impose de placer le modèle dans les mêmes états de contrainte que le "prototype" en grandeur réelle. Cette condition est satisfaite en soumettant un modèle réduit à l'échelle $1 / \mathrm{n}$ à une accélération centrifuge de $\mathrm{n}$ fois la pesanteur terrestre (ng).

Les applications à la géotechnique sont apparues tardivement car il a fallut développer de grandes centrifugeuses pour travailler à une échelle de réduction raisonnable. Ces applications se multiplient aujourd'hui avec les possibilités 
croissantes d'instrumentation des modèles et d'intervention en cours de centrifugation.

La caractérisation des sols en cours de centrifugation est possible depuis plusieurs années au moyen de dispositifs dits "embarqués". A ce jour existent des mini pénétromètres statiques et des mini scissomètres qui reproduisent les essais in situ. La miniaturisation a été facilitée par la simplicité même de ces appareils, à la différence du pressiomètre. La détermination de $\mathrm{Em}$ et $\mathrm{Pl}$, souvent nécessaire à toute étude approfondie, s'effectue actuellement par corrélation avec la résistance de pointe qc de l'essai pénétrométrique ou la cohésion $\mathrm{Cu}$ de l'essai scissométrique. Une mesure directe serait préférable mais nécessite le développement d'une sonde pressiométrique miniature.

\subsection{La mini pressiométrie}

La sonde de mesure du pressiomètre Ménard, de diamètre $\phi=60 \mathrm{~mm}$ et de longueur $\mathrm{l}=360 \mathrm{~mm}$, est équipée de cellules de garde. Pour en simplifier l'utilisation ou pour d'autres applications, des pressiomètres aux dimensions plus modestes sont apparus :

- Le pressiomètre pour chaussées de J.L.Briaud $(\phi=31 \mathrm{~mm}, 1=230 \mathrm{~mm})$ [1].

- Le modèle Apagéo $(\phi=25 \mathrm{~mm}, 1=285 \mathrm{~mm})$.

- Le mini pressiomètre pour cellules triaxiales de W.F.Anderson, J.C.Pyrah et F.Haji Hali $(\phi=25 \mathrm{~mm}, 1=150 \mathrm{~mm})$ [2].

Les massifs de sol centrifugés connaissent de forts gradients et la "ponctualité" de l'essai cautionne la qualité des résultats. L'utililisation en centrifugeuse impose une faible longueur de sonde et rend les mini pressiomètres existants inadaptés.

Les travaux de développement du prototype ont commencé au LCPC en 1989 avec G.Moreau [3], ont été repris en 1996 par M.Berthelot et J.Dejour [4], et poursuivis en 1997 par O.Beckerich [5].

\section{Le mini pressiomètre}

\subsection{Description de la sonde}

Les dimensions retenues de la sonde sont un diamètre $\phi=10 \mathrm{~mm}$ pour une longueur $1=20 \mathrm{~mm}$.

La mini sonde simule ainsi la sonde standard pour des modèles réduits au $1 / 20^{\circ}$ et centrifugés à $20 \mathrm{~g}$. Le diamètre n'a pu être davantage réduit pour des raisons mécaniques. L'élancement de la sonde est donc de 2, contre 6 pour une sonde Ménard équipée de cellules de garde. Toutefois, les essais ont montré que l'hypothèse d'une expansion cylindrique restait très raisonnable $(\S 3)$.

La sonde est équipée d'une membrane en latex naturel de $6 / 10^{\circ} \mathrm{mm}$ d'épaisseur, maintenue de part et d'autre par deux entretoises (figure 1). Elles 
assurent également l'étanchéité par pincement de la membrane. Le diamètre intérieur du corps de sonde permet le passage du capteur de pression. Un tube flexible en polyamide relie la sonde au CPV. L'inertie de la membrane est inférieure à $30 \mathrm{kPa}$. Le fonctionnement est satisfaisant jusqu'à $4000 \mathrm{kPa}$.

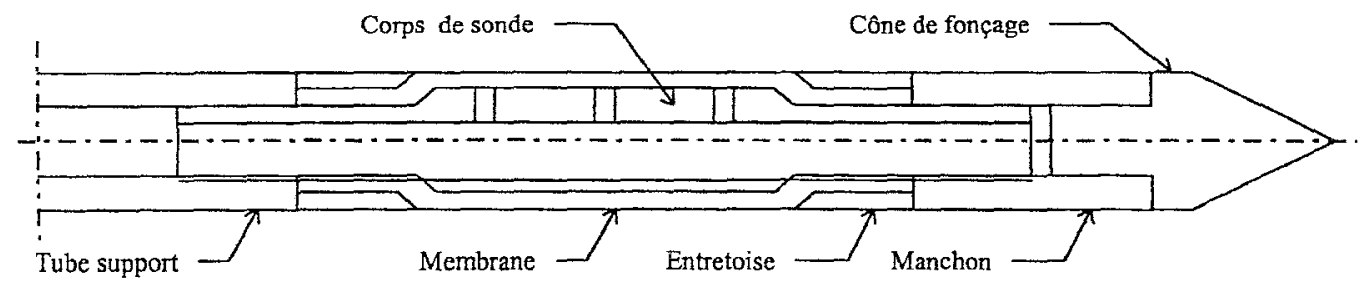

Figure 1 : coupe de la sonde.

Actuellement, la mise en place de la sonde dans le sable s'effectue par fonçage manuel. Dans l'argile et les limons, il faut réaliser un forage préalable. La fragilité de la membrane utilisée dans le présent prototype impose de mener ces opérations à $1 \mathrm{~g}$.

\subsection{Description du contrôleur pression-volume}

Par souci de simplification, le contrôleur pression-volume (CPV) a été conçu pour un fonctionnement à volume contrôlé.

Un vérin électrique à courant continu $(0,24 \mathrm{~V})$ pousse le piston d'un vérin hydraulique, dont le fonctionnement est analogue à celui d'une seringue (figure 2). Il met ainsi en pression le circuit d'eau.

La mesure de volume se fait par un capteur de déplacement du piston. La mesure de pression s'effectue au moyen d'un capteur miniature Entran (diamètre extérieur de $3.2 \mathrm{~mm}$ ) situé dans la sonde. L'évolution du chargement, d'une durée moyenne d'une minute, est suivie en temps réel.

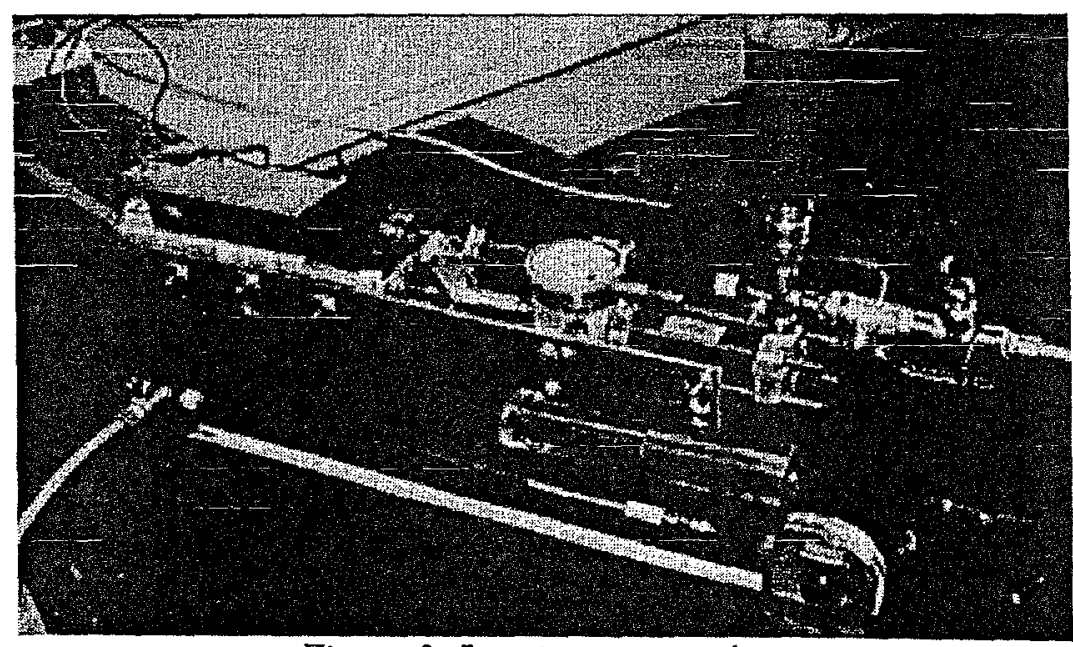

Figure 2: Le mini pressiomètre. 


\section{Déformation de la sonde}

\subsection{Géométrie de l'expansion}

Nous avons mené deux types d'expériences afin de quantifier le défaut de cylindricité d'expansion de la membrane, dû au faible élancement de la sonde.

La première a consisté à positionner la sonde dans un tube transparent de diamètre intérieur $12 \mathrm{~mm}$, et à effectuer une montée en pression.

On observe dans un premier temps une expansion sphérique de la membrane. Le contact de la partie centrale avec le tube est établi à $25 \mathrm{kPa}$ (figure 3). A $100 \mathrm{kPa}$, la totalité de la membrane est plaquée contre le tube (figure 4). On commence alors à observer un écrasement du latex qui vient combler les aspérités du tube, sur toute la longueur de la sonde. Dès $100 \mathrm{kPa}$, la régularité de l'expansion est donc satisfaisante.
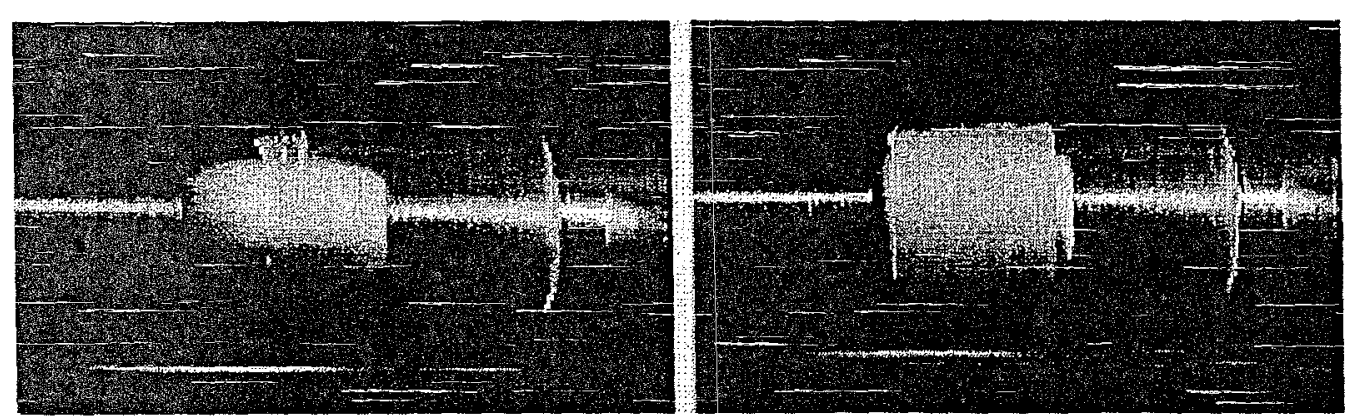

Figures 3 et 4 : Expansion de la membrane.

La seconde expérience a consisté à mener plusieurs essais pressiométriques dans un massif d'argile, à $1 \mathrm{~g}$. La découpe du massif a ensuite permis de dégager les empreintes laissées par la membrane (figures 5 et 6).
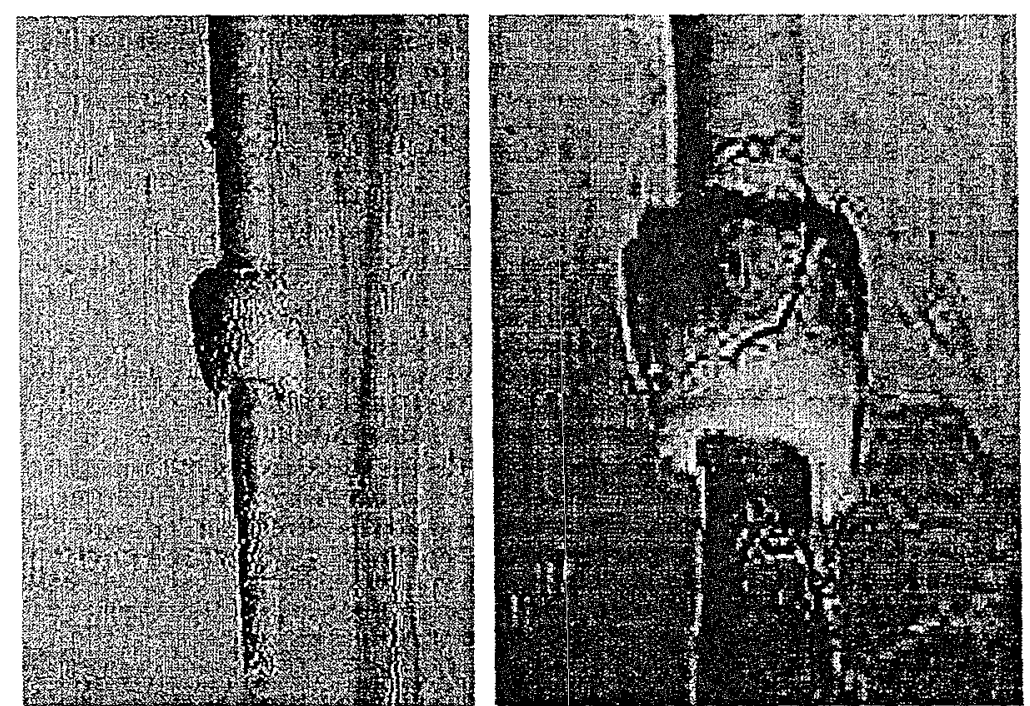

Figures 5 et 6 : empreintes des essais pressiométriques. 
Le défaut de cylindricité dû à l'absence de cellules de garde est minime et ne concerne que $10 \%$ environ de la longueur de la sonde.

Les volumes injectés sont élevés par rapport au volume initial de la sonde. En moyenne, le diamètre résiduel de la cavité a doublé, soit un volume final 4 à 6 fois supérieur au volume initial.

\section{2 Étalonnage dans le tube rigide}

Les premiers essais ont montré l'importance des déformations internes de l'appareil, qui ne sont pas négligeables devant les faibles volumes injectés au cours d'un essai pressiométrique.

Plusieurs essais de calibrage ont été menẻs en insérant la sonde dans un tube d'acier ajusté dont la déformation est négligeable. La constante de compressibilité "a" s'établit à $0.420 \mathrm{~mm} 3 / \mathrm{kPa}$ sur toute la plage de pression.

Ce sont principalement les durites en polyamide qui se dilatent. Le volume qu'elles libèrent est de l'ordre de $15 \%$ du volume total injecté. Cette correction n'est pas véritablement gênante puisque l'appareil reste dans le domaine des déformations élastiques.

\section{Essais en modèles centrifugés}

\subsection{Programme expérimental}

Une série d'essais pressiométriques a été menée dans un conteneur de sable de Fontainebleau réalisé par pluviation, d'une densité moyenne de $16.14 \mathrm{kN} / \mathrm{m} 3$ pour un indice de densité de 0.70 . Le mini pressiomètre a été testé à $10,20,30$ et $40 \mathrm{~g}$.

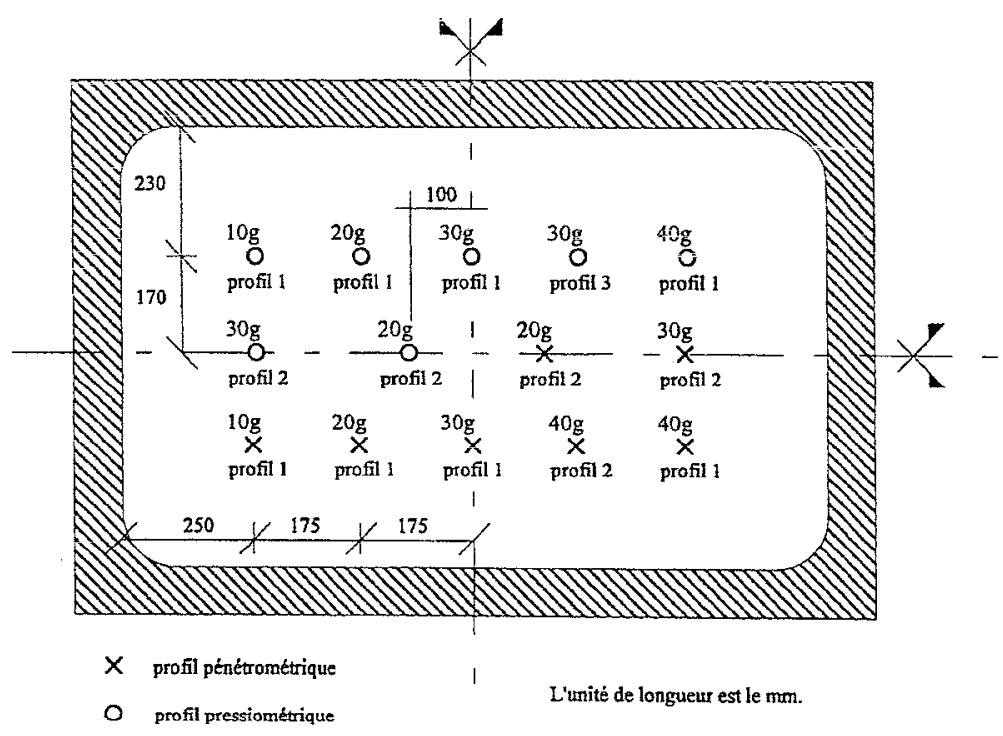

Figure 7 : Implantation dans le conteneur. 
Plusieurs profils pressiométriques ont été réalisés à chaque niveau d'accélération, constitués de trois essais aux profondeurs de $-90,-180$ et $-270 \mathrm{~mm}$. Un essai au mini pénétromètre a systématiquement été mené parallèlement, afin de permettre une corrélation (tableau 1 et figure 7).

Tableau 1 : Récapitulatif des essais

\begin{tabular}{ccc}
\hline & $\begin{array}{c}\text { nombre de profils } \\
\text { pressiométriques }\end{array}$ & $\begin{array}{c}\text { nombre de profils } \\
\text { pénétrométriques }\end{array}$ \\
\hline $10 \mathrm{~g}$ & 1 & 1 \\
$20 \mathrm{~g}$ & 2 & 2 \\
$30 \mathrm{~g}$ & 3 & 2 \\
$40 \mathrm{~g}$ & 1 & 2 \\
\hline
\end{tabular}

\subsection{Courbe type et caractéristiques pressiométriques}

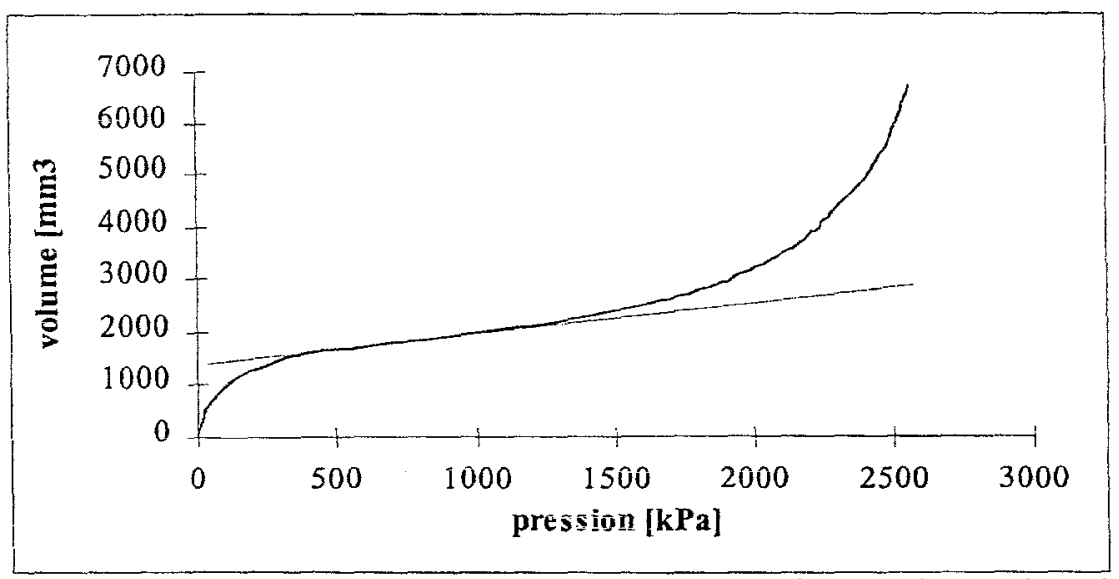

Figure 8: Courbe pressiométrique type (sable de fontainebleau dense).

La figure 8 montre un exemple de résultat d'essai à $20 \mathrm{~g}$ et à une profondeur modélisée de $8,10 \mathrm{~m}$. On retrouve l'allure de la courbe pressiométrique d'un essai à sonde standard. La partie pseudo élastique apparait nettement, permettant un calcul fiable du module pressiométrique. La détermination de la pression limite en considérant le doublement de volume de la sonde de mesure ne peut s'appliquer au mini pressiomètre, ce point de la courbe appartenant souvent à la partie élastique. $\mathrm{La}$ pression limite est donc lue sur la courbe, au risque d'une surévaluation, avec un maximum correspondant à 5 fois le volume initial. Un nouveau critère devra éventuellement être recherché. La pression de fluage pf est en revanche difficile à estimer, ce qui rejoint les observations faites sur les courbes de l'essai standard.

\subsection{Profils pressiométriques}

Les profils pressiométriques, en données modèle, montrent une évolution linéaire du module et de la pression limite en fonction de la profondeur (figure 9).

Les écarts entre deux essais réalisés à la même profondeur et à la même accélération sont limités. Ils s'expliquent en partie par des effets de bords (proximité des parois du conteneur). 

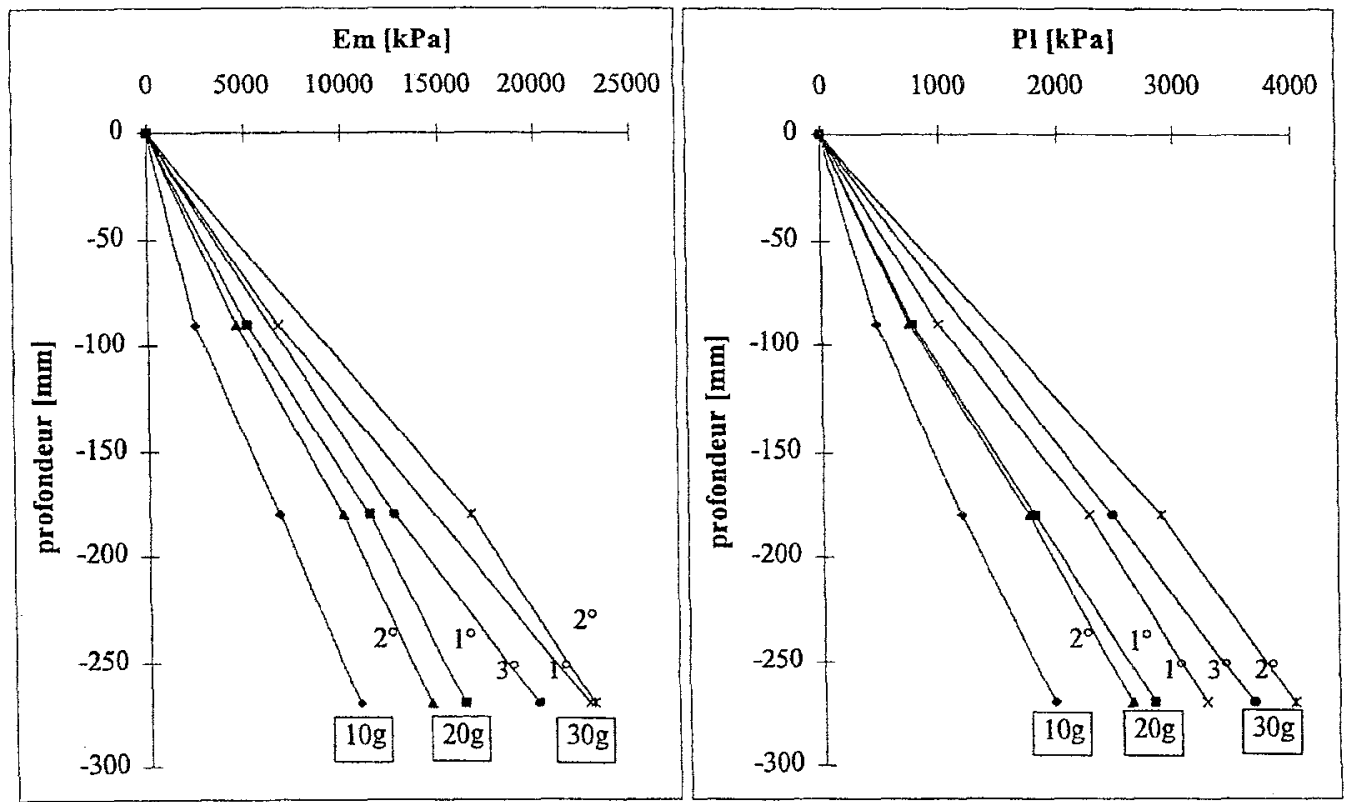

Figure 9 : Profils pressiométriques (sable de Fontainebleau dense).

4.4 Rapport $\frac{E m}{p l}$ et corrélations pressiomètre/pénétromètre

Le tableau 2 donne les valeurs des rapports $\mathrm{Em} / \mathrm{pl}$, qc/pl, et Em/qc déduites des essais au mini pressiomètre et mini pénétromètre (pointe de diamètre $12 \mathrm{~mm}$ ) en centrifugeuse. Ces rapports ne dépendent pas de l'accélération centrifuge.

Tableau 2 : Moyenne par niveau d'accélération des rapports $\frac{E m}{p l}, \frac{q c}{p l}$ et $\frac{E m}{q c}$.

\begin{tabular}{ccccccc}
\hline & $10 \mathrm{~g}$ & $20 \mathrm{~g}$ & $30 \mathrm{~g}$ & $40 \mathrm{~g}$ & moyenne & sonde standard [6] \\
\hline$\frac{E m}{p l}$ & 5.5 & 6 & 6 & 5.4 & 5.7 & 5 à 7 (sol sous consolidé) \\
$\frac{q c}{p l}$ & 6.5 & 6.7 & 6.9 & & 6.7 & 7 à 12 (sol normalement consolidé) \\
$\frac{E m}{q c}$ & 0.88 & 0.90 & 0.88 & & 0.88 & 9 \\
\hline
\end{tabular}

La valeur moyenne de $5.7 \mathrm{du}$ rapport $\mathrm{Em} / \mathrm{pl}$ est plus faible que celle des rapports obtenus en essais in situ. L'ordre de grandeur est toutefois conservé.

Une explication possible est la surévaluation de la pression limite (\$4.2). Si sa valeur exacte était inférieure de $30 \%$, le rapport $\mathrm{Em} / \mathrm{pl}$ dépasserait 8 , et correspondrait, selon les fourchettes des essais in situ, à l'état de consolidation effectif du massif.

Le rapport qc/pl mérite les mêmes commentaires. Le rapport Em/qc est par contre assez proche de la fourchette habituelle. 


\section{Conclusions}

Le mini pressiomètre doit permettre une mesure directe en cours de centrifugation de modules pressiométriques et de pressions limites nécessaires aux études sur modèles réduits. Ces paramètres sont actuellement obtenus par corrélations avec les résultats d'essais au mini pénétromètre.

Le prototype actuel, étanche et calibré, est mécaniquement en état de fournir ces mesures. L'étude de la déformation de la sonde a montré une cylindricité d'expansion satisfaisante et un fonctionnement analogue à celui d'une sonde Ménard.

L'appareil a permis de mener les tout premiers essais pressiométriques en centrifugeuse. Sa plage d'utilisation est de $1 \mathrm{~g}$ à $50 \mathrm{~g}$, avec une optimisation à $20 \mathrm{~g}$.

Les courbes et profils pressiométriques des essais modélisant des profondeurs de $0.90 \mathrm{~m}$ à $8.10 \mathrm{~m}$ sont fidèles à ceux des essais in situ. L'ordre de grandeur des mođules et pressions limites est conservé.

il reste à développer une méthode standãdisée de détermination de la pression limite et à préciser les corrélations avec le mini pénétromètre.

Le fonçage à $1 \mathrm{~g}$ et la cicatrisation du soì avec la montée en $\mathrm{g}$ rendent le remaniement du sol négligeable. Cependant le soin apporté à la mise en place de la sonde cautionne la qualité du résultat. A terme, les essais au mini pressiomètre devraient être menés à l'aide du téléopérateur embarqué sur la centrifugeuse.

La prochaine étape de développement est le rapprochement des essais pressiométriques modèle et prototype afin de déterminer l'éventuel effet d'échelle.

Certaines applications in situ sont aussi envisageables après avoir résolu le problème de la mise en place dans un sol déjà consolidé. Elles concernent les cas où le pressiométre standard est trop volumineux, trop lourd d'emploi (essais en offshore) ou trop perturbateur (au voisinage des structures).

\section{Références bibliographiques :}

[1] Briaud, $\mathrm{JL}$ et Shield, DH. (1981). "Pressuremeter tests at very shallow depht". Journal of Geotechnical Engineering, Vol $107 \mathrm{~N}^{\circ} \mathrm{GT} 8$.

[2] Anderson, WF, Pyrah, IC et Haji Ali, F (1987). "Rate effects in pressuremeter tests in clays". Journal of Geotechnical Engineering, Vol 113 $\mathrm{N}^{\circ} 11$.

[3] Moreau, G (1989). "Un mini pressiomètre au banc d'essai". Rapport de DEA. École Centrale de Nantes.

[4] Berthelot, $M$ et Dejour, J (1996). Projet de fin d'études. École Navale.

[5] Beckerich, O (1997). "Développement d'un mini pressiomètre-Application aux modèles réduits centrifugés". Rapport de DEA. École Centrale de Nantes.

[6] Cassan, M (1978). "Les essais in situ en mécanique des sols", Volume 1. Eyrolles Éditions. 